\title{
Dissecting the mechanisms of EIN3-dependent regulation of ethylene response in Arabidopsis thaliana
}

\author{
E.V. Zemlyanskaya ${ }^{1,2 *}$, D.Y. Oshchepkov ${ }^{1}$, V.V. Mironova ${ }^{1,2}$, V.G. Levitsky ${ }^{1,2}$ \\ ${ }^{1}$ Institute of Cytology and Genetics SB RAS, Novosibirsk, Russia \\ ${ }^{2}$ Novosibirsk State University, Novosibirsk, Russia \\ *e-mail: ezemlyanskaya@bionet.nsc.ru
}

Key words: transcription factor, epigenetic regulation, ETHYLENE-INSENSITIVE3, EIN3 binding site (EBS), ChIP-seq, RNA-seq

Motivation and Aim: The plant hormone ethylene regulates numerous developmental processes and stress responses [1]. Ethylene signaling proceeds via a linear pathway, which activates EIN3 transcription factor [2]. EIN3 influences gene expression upon binding to a specific sequence in gene promoters. However, in many cases EIN3 binding to gene promoter is not enough to trigger transcriptional response [3]. Here we perform whole genome bioinformatics study to dissect the factors essential for EIN3 functioning. Methods and Algorithms: We extract EIN3 binding regions (EBRs) from publicly available ChIP-Seq data on EIN3 binding in Arabidopsis thaliana [3] and use RNAseq data on ethylene-induced transcriptomes [3] to determine ethylene responsiveness of corresponding genes. We further investigate the impact of DNA-binding context, its position relative TSS and epigenetic status on the ethylene sensitivity of genes bound by EIN3. We use previously published genome-wide map of nine chromatin states in A. thaliana [4] to characterize EIN3 binding regions with respect to the epigenetic status. Results: The analysis of ChIP-seq data showed bimodality of distribution of EIN3 binding regions in gene promoters. We found that the implicit distal peak was associated with a specific chromatin state (referred to as chromatin state 4 in the primary source), which was just poorly represented in the pronounced proximal peak. EIN3 binding regions corresponding to the chromatin state 4 were significantly associated with ethylene response, unlike the others representing the overwhelming majority of EBRs related to the explicit proximal peak. Moreover, we found that specific EIN3 binding sequences predicted with previously described model were enriched specifically in the EBRs mapped to the chromatin state 4, but not to the rest ones [5].

Conclusion: These results allow us to conclude that the interplay of genetic and epigenetic factors might cause the distinct modes of EIN3 regulation.

Acknowledgements: The work was supported by Russian Foundation for Basic Research and the government of Novosibirsk region, grant No. 18-44-540039.

References

1. Abeles F.B. et al. (1992) Ethylene in plant biology. San Diego: Acad. Press.

2. Merchante C. et al. (2013) Ethylene signaling: simple ligand, complex regulation. Curr. Opin. Plant Biol. 16:554-560.

3. Chang K.N. et al. (2013) Temporal transcriptional response to ethylene gas drives growth hormone crossregulation in Arabidopsis. Elife. 2:e0675.

4. Sequeira-Mendes J. et al. (2014) The functional topography of the Arabidopsis genome is organized in a reduced number of linear motifs of chromatin states. Plant Cell. 26:2351-2366.

5. Zemlyanskaya E.V. et al. (2017) The interplay of chromatin landscape and DNA-binding context suggests distinct modes of EIN3 regulation in Arabidopsis thaliana. Front. Plant. Sci. 7:2044. 